\title{
La inteligencia emocional y el rendimiento académico en los estudiantes de la Facultad de Ciencias de la Salud de la Universidad Nacional Toribio Rodríguez de Mendoza de Amazonas, Chachapoyas 2018.
}

\section{Emotional intelligence an academic perfomance in the students of the Faculty of Health Sciences of the Toribio Rodriguez de Mendoza National University of Amazonas, Chachapoyas 2018}

\author{
Erwin Alberto Cabrera Soto ${ }^{1}$
}

\begin{abstract}
RESUMEN
El trabajo de investigación tuvo como objetivo determinar la relación que existe entre la inteligencia emocional y el rendimiento académico en los universitarios de las distintas escuelas profesionales de salud. El tipo de investigación fue sustantiva correlacional, la muestra del estudio estuvo conformada por 142 alumnos, utilizando el instrumento ICE-Bar On (Emotional Quotient Inventory), adaptado por Nelly Ugarriza en el Perú (2005), constituido por 133 ítems que ofrecen cinco puntuaciones diferentes en base a la escala de Lickert. Los datos recolectados fueron introducidos en una base de datos, para ser procesados mediante el paquete estadístico computacional SPSS Versión 19. Los resultados evidenciaron que en aspectos generales no existe relación significativa entre la Inteligencia Emocional y el Rendimiento Académico ya que es débil positiva y no significativa porque en sus componentes intrapersonal, interpersonal, adaptabilidad la relación es débil, directa y no significativa y en los componentes manejo de tensiones y estado de ánimo es débil inversa y no significativa, concluyendo finalmente que la presencia de una no predice a la otra de manera confiable y significativa.
\end{abstract}

Palabras clave: Inteligencia emocional, rendimiento académico.

\begin{abstract}
The objective of the research work was to determine the relationship between emotional intelligence and academic performance in the university students of the different health professional schools. The type of research was correlational, the study sample consisted of 142 students, using the instrument ICE-Bar On (Inventory of emotional quotient), adapted by Nelly Ugarriza in Peru (2005), consisting of 133 items that you Five Five Different at the base of the Lickert scale. The data collected were entered into a database, to be processed using the statistical package SPSS Version 19. The results showed that in the general aspects there are no relationships between Emotional Intelligence and Academic Performance and that it is weak positive and not significant because in its intrapersonal, interpersonal, and adaptability components the relationship is weak, direct and not significant, and in the components Tension and mood management is weak inverse and not significant, finally concluding that the presence of a person does not have a reliable and significant basis.
\end{abstract}

Keywords: Emotional intelligence, academic performance. 


\section{INTRODUCCIÓN}

En la actualidad el estudiante universitario para que logre el éxito académico, tiene que desarrollar habilidades académicas que sean flexibles y dinámicas para adaptarse de manera continua a los múltiples cambios de una sociedad globalizada, y esta a su vez origina en el estudiante alteraciones en su estado emocional, debido a la presión de los estudios, llevándolo al borde de sus propios límites físico y psíquicos (López, 2008).

Por tanto, ya no basta que el universitario sólo analice e integre información curricular, sino que también se convierta en parte activa de su proceso formativo integrándose al mismo tiempo con sus padres y docentes, aspecto que implica la regulación de emociones, habilidad que, de ser desarrollada por el alumno, influenciaría positivamente en su rendimiento académico.(Newsone,2000)

En ese sentido la universidad descuida el trabajo emocional del estudiante y profesor en lo académico, por ejemplo Mayer y Salovey (2007), refiere que los investigadores, docentes, administraciones educativas e incluso la misma sociedad en general, han ido haciéndose más y más conscientes de la importancia por velar una educación integral que considere los aspectos emocionales, y que no se limite a la potenciación, casi exclusiva de las habilidades cognoscitivas y lingüísticas.

Sánchez, et al. (2007) señala que el ambiente amistoso estimula la confianza y la espontaneidad en el estudiante, que va a desarrollar toda su potencialidad, por el contrario, un ambiente donde impera la amenaza y el miedo, va a favorecer la aparición de actitudes dependientes por parte de los estudiantes.

Taush (1999), demostró que la personalidad del maestro es fundamental en el desarrollo y progreso de los estudiantes. Encontró que muchos maestros que tenían conocimientos científicos acerca de cómo tratar a sus estudiantes, no podían poner en práctica dichas enseñanzas, pues su personalidad se lo impedía, en cambio Goleman, (1995) señala que la inteligencia emocional se basa en los aspectos emocionales de la vida de las personas, no cognitivas, que hacen que las clásicas inteligencias se incrementen gracias a su poder modulador. Es importante educar la inteligencia emocional, porque va a permitir resolver problemas de la vida, específicamente la labor academica. Tanto es así que tantos padres como docentes consideren fundamental el trabajo en inteligencia emocional en el ámbito educativo, dado que el dominio de habilidades de este tipo de inteligencia sería beneficioso. Extremera y Fernández-Berrocal (2004) manifiesta por ejemplo que la inteligencia emocional intrapersonal, experimentan con claridad los sentimientos propios y poder reparar los estados emocionales negativos, influirá decisivamente sobre la salud mental de los estudiantes y este equilibrio emocional, a su vez está relacionado y afecta al rendimiento académico final.

En lo referente al rendimiento académico se indica que es afectado por una serie de variables que van desde estado de ánimo diversos, hasta la alimentación o el tiempo y dedicación al estudio, es menester enfatizar queuno de los mejores elementos que le sirven de estímulo y motivación al estudiantado en el proceso enseñanza aprendizaje es el docente, es en esta relación académica que se pone en juego las emociones de ambos, destacando la del estudiante que está en pro de lograr el desarrollo total de su personalidad. Cada estudiante presenta varias dimensiones emocionales positivas que le va permitir afrontar desavenencias o conflictos en sus interrelaciones, pero muchos estudiantes presentan dificultades para solucionar sus problemas de índole emocional, personal y, que no le va a permitir lograr un rendimiento académico óptimo en sus estudios superiores.

En ese sentido, el objetivo general de esta investigación fue determinar la relación entre la inteligencia emocional y el rendimiento académico de los estudiantes de la Facultad de ciencias de la salud de la UNTRM, de Amazonas. 2018.

\section{MATERIAL Y MÉTODO}

La investigación planteada se de tipo sustantiva correlacional y el diseño aplicado es correlacional básico no experimental transversal. El método empleado es el inductivo y deductivo, empleando el coeficiente de correlación de Spearman, y su respectiva prueba estadística. Para todas las pruebas estadísticas, se eligió un valor de 0.05 para el nivel de significación.

La población universal estuvo constituida por 604 estudiantes de las carreras profesionales de Psicología, Estomatología, Medicina, y Enfermería del I al X ciclo de la Facultad de Ciencias de la Salud del Semestre 2018-I. La muestra fue de 230 estudiantes. El tipo de muestreo utilizado fue el estratificado por afijación proporcional en relación a los ciclos, así como el muestreo aleatorio simple de cada ciclo. 
Técnicas e instrumentos de recolección de datos: La técnica utilizada fue la encuesta y el instrumento de recolección de datos, el cuestionario- Inventario Emocional de BarOn (I-CE),

BarOn (1997. Citado por Ugarriza et al. 2005) el instrumento consta de 133 ítems que ofrecen cinco puntuaciones diferentes referidos a componentes del cociente emocional, estos son intrapersonal, interpersonal, manejo de estrés, adaptabilidad y estado de ánimo, y estos a su vez contienen 15 subcomponentes. Para cada ítems hay cinco posibles respuestas que van desde "siempre" a "nunca" en una escala tipo likert de 1 a 5 puntos. Y es usado porque tiene una medición confiable y valida.

En cuanto al rendimiento académico se trabajó con el puntaje promedio de calificación cuantitativa final de una asignatura de especialidad, obtenido por los estudiantes de las diferentes especialidades.

\section{RESULTADOS}

En la figura 1 se observan los puntajes promedio de todos los componentes del cociente emocional, presentando el mayor puntaje el componente Intrapersonal (114.57) y promedio menor en el componente Manejo de Estrés (48.69)

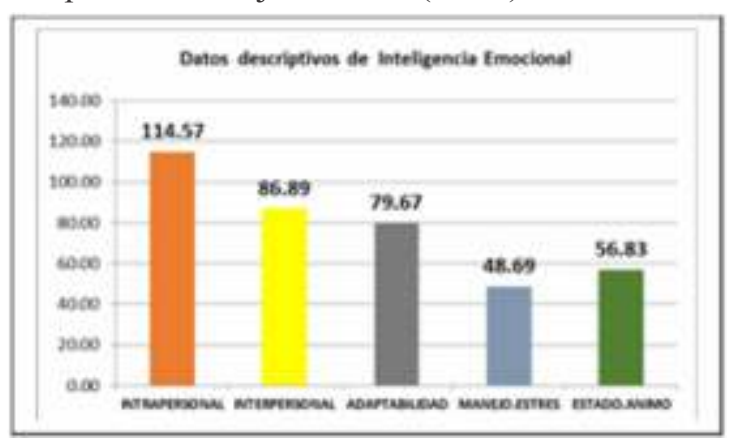

Figura 1. Puntajes promedio de los componentes de inteligencia emocional

En lo concerniente a la figura $\mathrm{N}^{\circ} 2$, las frecuencias correspondientes al nivel del componente Intrapersonal del cociente emocional, se encontró que mayor frecuencia el nivel correspondiente a promedio $(59.6 \%)$ y en menor frecuencia el nivel bajo $(0.4 \%)$.

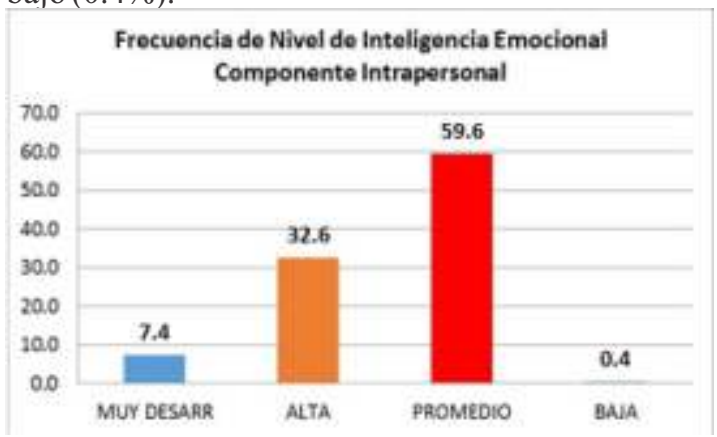

Figura 2: Niveles del componente Intrapersonal del cociente emocional.

En lo que se refiere a la figura $\mathrm{N}^{\circ} 3$ las frecuencias correspondientes al nivel del componente Interpersonal del cociente emocional se encontró con mayor frecuencia el nivel correspondiente a promedio $(82.4 \%)$ y en menor frecuencia el nivel marcadamente alta $(0.7 \%)$.

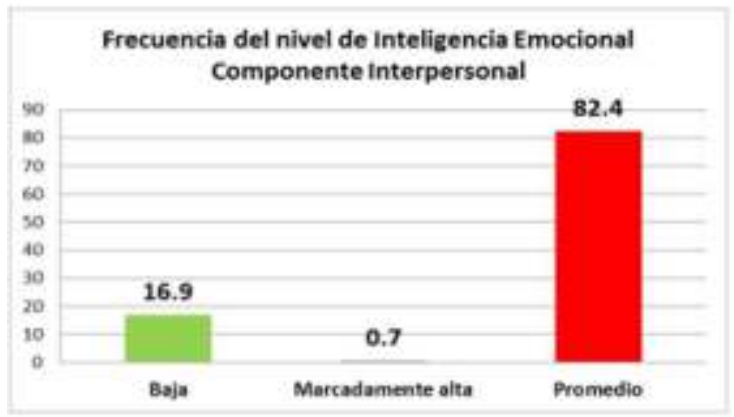

Figura 3: Niveles del componente interpersonal del cociente emocional

En relación a la figura $\mathrm{N}^{\circ} 4$ las frecuencias correspondientes al nivel del componente adaptabilidad se encontró con mayor frecuencia los niveles correspondientes a alto y promedio $(53.5 \%) \mathrm{y}$ en menor frecuencia el nivel bajo $(2.1 \%)$.

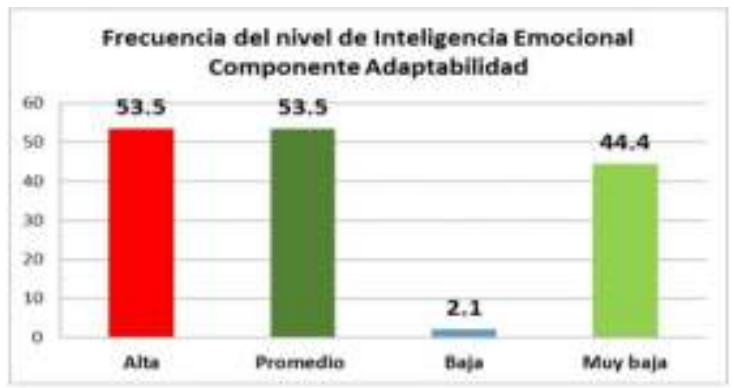

Figura 4: Niveles del componente adaptabilidad del cociente emocional

En relación a la figura $\mathrm{N}^{\circ} 5$ las frecuencias correspondientes al nivel del componente manejo del estrés del cociente emocional se encontró que el nivel muy bajo es quien presenta la totalidad de frecuencias $(99.6 \%)$

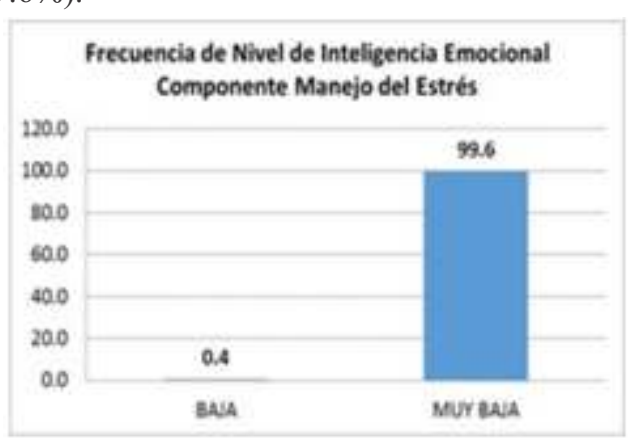


Figura 5: Niveles del componente manejo de estrés del cociente emocional

Dentro de los objetivos específicos de esta investigación se busca precisar el nivel de componente animo general del cociente emocional, como se aprecia en la figura $\mathrm{N}^{\circ} 6$ las frecuencias correspondientes al nivel del componente animo general del cociente emocional se encontró que el nivel muy bajo es quien presenta la totalidad de las frecuencias $(97.8 \%)$.

\section{Frecuencia de Nivel de Inteligencia Emocional} Componente Estado de Animo General

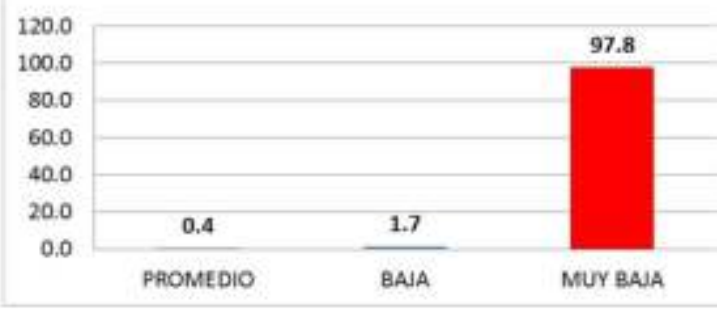

Figura 6: Niveles del componente animo general del cociente emocional

En este gráfico de frecuencias de notas, se aprecia que la nota 13 y 12 cuentan con una mayor frecuencia (36.5 y 30.9\% respectivamente), los más bajos son las notas de 17 y 11 y 16 ( $0.4 \%$ y $2.2 \%$ cada uno).

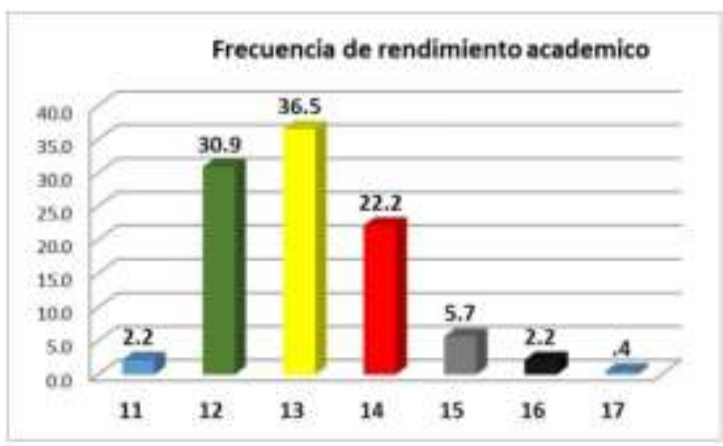

Figura 8. Puntaje promedio de la frecuencia de notas de la muestra.

\section{DISCUSIÓN}

Los resultados encontrados entres estas dos variables, inteligencia emocional y rendimiento académico, guardan por un lado una relación directa débil, directa y no significativa con los componentes de intrapersonal, interpersonal y adaptabilidad, en tanto que, en el estado de ánimo y manejo de tensión, la relación es débil inversa y no significativa, y, siendo la correlación en general débil positiva y no significativa, donde la presencia y dirección de una no predice a la otra de una manera confiable y significativa, no existiendo correlación entre ambas variables y por alcanzar un coeficiente muy bajo y no significativo mucho mayor al 0.05 .

En cuanto a los componentes intrapersonal e interpersonal sus resultados nos indican que en el primero, medianamente se sienten bien consigo mismo, expresar sus sentimientos y confianza en la realización de sus ideas, igual resultado se logra en el segundo debido a que los estudiantes regularmente desarrollan habilidades sociales

En cuanto al manejo de tensión se presenta un coeficiente de correlación negativo, pues esinferior al -0.20 , presentando una escasa resistencia al estrés y propensos a perder el control emocional inmediatamente, así mismo que, si bien entre los componentes de adaptabilidad, estado de ánimo y el rendimiento académico, se producen relaciones positivas, estas no son significativas.

Este resultado en general podría explicarse que más allá de un cierto nivel intelectual, sean otros factores de tipo personal los que mantengan algún tipo de relación, al menos con el logro académico.

Extremera, Fernandez-Berrocal (2004), en sus investigaciones sobre inteligencia emocional y rendimiento académico encontraron resultados contradictorios en todos los niveles educativos, de igual manera Hernández (2010) en su planteamiento de los moldes cognitivo-afectivo como modelo de la inteligencia emocional ha llegado a resultados similares. Probablemente debido a las exigencias de un alto rendimiento en los exámenes de admisión y que continua en sus estudios académicos en desmedro del desarrollo de la inteligencia emocional, todo indica que habrá que relacionarla con otras variables para obtener resultados más concluyentes.

Por otra parte, la aparición de correlaciones prácticamente nulas, ninguna de ellas Significativa, indica la independencia de ambas variables, lo cual coincide con los hallazgos de otros citados $\quad \begin{array}{lll}\mathrm{p} & \mathrm{r}\end{array}$ Extremera, Fernandez- Berrocal (2004). De igual manera Bastian, Burns, Netellbeck (2005), Barchard (2003), Chico (1999), Newsone (2000), Martinez (2010) y Sanchez, Rodriguez y Padilla (2007) utilizaron escalas de reporte de diferentes instrumentos de medición no encontrando relaciones significativas entre las dos variables,en donde los universitarios presentaron baja capacidad de expresión, sentir emociones de forma precisa, no podían comprender y manejar adecuadamente sus emociones no concordando con sus logros académicos.

Así mismo Parker (2003), hallo divergencias en 
función como se operacionalizo la variable rendimiento académico cuando examino la relación, utilizo una escala autoparte basado en la teoría de la inteligencia emocional de BarOn, encontrando correlación, pero no en las subescalas demostrando una vez más su inconsistencia.

Jiménez y López-Zafra (2007) dieron a conocer que la relación de ambas variables son inconsistentes debido a que no existe consenso en la definición operacional del constructo y la metodología tan diversa que presentan los estudios, de igual modo Newsome (2000) indica que existen varias prueba que miden la inteligencia emocional como el TMMS-24, el TEIQ, el MSCEIT y el BarOn ICE, que no miden rigurosamente, ya que sus resultados son limitados, inconsistentes y contradictorios.

En cuanto al rendimiento académico, Artunduaga(2010), demostró que es necesario considerar en esta relación, variables como el estudiante, profesor, contexto, metodología, estrategias, medios y sistemas de evaluación que mejor describen y predicen el rendimiento académico. Adell (2006) amplio y reforzó las investigaciones anteriores al señalar que el rendimiento es un constructo multidimensional determinado por (inteligencia, motivación, personalidad, etc.) y en el que influyen numerosos factores, personales, familiares, entre otros. Finalmente los hallazgos de esta investigación se integran al cuerpo deevidencias que cuestionan la relación de ambas variables.

\section{CONCLUSIONES}

Contrariamente a lo esperado, no se produce ninguna relación significativa entre las variables inteligencia emocional y rendimiento académico. Siendo la correlación en general débil positiva y no significativa. Estos resultados se deben a que existe un desnivel entre los cinco componentes, debido a que mientras en los componentes intrapersonal, interpersonal y adaptabilidad presentan un nivel promedio; en estado de ánimo y estrés su nivel es muy bajo y en cuanto al rendimiento académico, el promedio de notas en general es mayormente regular. Todo esto nos indica que la correlación en los tres primeros es directa débil, directa y no significativa y en los dos últimos la relación es débil, inversa y no significativa.

Estos resultado podría verse explicado por el hecho de que se trata de estudiantes, nivel educativo, en el que dejan de aparecer en muchas ocasiones correlaciones entre inteligencia emocional y el rendimiento académico, debido a la selección de estudiantes y a otros factores de tipo personal, social y educativo. Así mismo es cuestión de revisar otras variables, investigar otros instrumentos psicológicos que sean adaptativos a ciertas realidades, ver el nivel educativo, la metodología, las estrategias, el docente y la familia, para que los resultados obtenidos sean más consistentes y no contradictorios.

\section{REFERENCIAS BIBLIOGRÁFICAS}

Adell, M. (2002). Estrategias para mejorar el rendimiento académico. Madrid: Ediciones Pirámide.

Artunduaga, M. (2009). Variables que influyen en el rendimiento.académico. Extraído el 16 de j u 1 i o d e $1 \quad 2013$ d e : http://www.slideshare.net/1234 5098776/variables- delrendimientoacadémico

Barchard, K. (2003). Does emotional intelligence assist in the prediction of academic success . Educational and psychological measurement. Recuperado el 19de diciembre del 2013 de: http://www.reme.uji.es

Baron, R. (2002). Baron emotional quotient short form (EQ-i: Short). Technical manual. Toronto: Multi-Healt Systems

Bastian, V.;Burns, N., Nettelbeck, T. (2005). Emotional intelligence predicts life skill, but not as well as personality and cognitive abilities. Personality and individual differences. Recuperado el 18 de diciembre del 2013 de http://www.reme.uji.es

Chico, E. (1999). Evaluación psicométrica de una escala de inteligencia emocional. Madrid: Boletín de Psicología

Extremera, N. (2007). El modelo de inteligencia emocional de Mayer y Salovey y su validez predictiva en muestra españolas. España: Universidad de Malaga.

Extremera, N., \& Fernández-Berrocal, P. (2004). La evaluación de la inteligencia emocional en la calidad de las relaciones interpersonales y la empatía de los estudiantes universitarios malagueños. Proyecto de innovación en la universidad. Innovación Educativa, Universidad de Málaga. Extraído el 24 de j u 1 i o $\quad$ d e $1 \quad 2013 \quad d$ e http://www.rieoei/deloslectores/46 5Extremera.pdf

Extremera, N. \& Fernández-Berrocal, P. (2004) El papel de la inteligencia emocional en el a lum nado: Evidencias empiricas. Revista Electrónica de Investigación Educativa. Consultado el 2 de diciembre de1o 2013 en: 
http://redie.uabc.mx/vo6no2/conte nidoextremera.htlm

Guil, R., Gil Olarte. (2007). Inteligencia emocionaly educación: desarrollo de competencias socioemocional. Manual de inteligencia emocional. Madrid: Piramide.

Guerra, L; Rivera, K \& Vega, N. (2010) La inteligencia emocional y el rendimiento académico en estudiantes de la Universidad Técnica Federico Santa María Chile. Extraído el 15 de junio del 2012 de: htpp://www.utfm.cl/programasde estudio/postgrado.htl

Goleman, D, (1995). La inteligencia emocional. Buenos Aires: Printing Books.

Jiménez, J (2010) Inteligencia emocional y rendimiento académico. España: Universidad de Jaén.

Jiménez, M y López-Zafra (2007). La Inteligencia emocional y rendimiento escolar: estado actual de la cuestión. Revista Latinoamericana de Psicologia.Colombia. Recuperado el 5 de julio del 2012: http://redalyc.ua.emex.mx/src/inicio/Ar/ Pdf/Red.jsp?

López, O. (2008). La inteligencia emocional y las estrategicas de aprendizaje como predictores del rendimiento académico en estudiantes universitarios. Tesis de Maestría: Universidad Nacional Mayor de San Marcos

Martínez, J (2010). Relación existente entre inteligencia emocional y rendimiento académico en estudiantes universitarios en un centro adaptado al espacio europeo de educación superior. Tesis de Maestría. Recuperado el 2 de octubre del 2013 de: http://www.eumed.net/rev/ced/18/jamg2 $\underline{\mathrm{htm}}$

Mayer, J., Caruso, D. \& Salovey, P. (2004). Inteligencia emocional: Teoría, resultados y consecuencias. San Francisco: Jossey-Bass.

Mayer, J. \& Salovey, P. (2007). ¿Qué es la inteligencia emocional? .Madrid: Editorial Pirámide

Mestre, J.M y Guil, R. (2004). La Inteligencia emocional. Madrid: Centro de Estudios Ramón Areces.Núñez, J. (2005) Relevancia de la inteligencia emocional e $n$ e 1 archivement académico de los estudiantes universitarios. Granada: España Simposio: Inteligencia emocional y modelos de metal en el bienestar subjetivo en el logro. Extraído el 5 de Mayo del 2012 de: hppt:/www.esp2005.com/horary. asp
Parker, J., Summerfeldt, L., Hogan, M, y Majeski, S. ( 20003 ). Emotion al intelligence and academic success: examining the transition from high school to university. Vancouver: Annual meeting of the Canadian psychological association. Recuperadoel6de junio de12012 de http://www.trentu.ca/academic/ psychology/jparker/keefer pre s maestro. México: Revista de Educación.

Pérez, N., Castejón, J. (2000). La inteligencia emocional como predictor del rendimiento académico en estudiantes universitarios. Madrid: Editorial Pirámide.

Sánchez, M.; Rodríguez, N. \& Padilla, M. (2007). Relación entre La inteligencia emocional y el rendimiento académico en los estudiantes de la facultad de psicología de la universidad autónoma de Nuevo león. Tesis de Maestría. R e c u p e r a d o e 115 d e j u 1 i o d e 120012 d e : http://www.academia.edu/296782 1/ La Inteligencia esta

Taush, R. (1999). El fenómeno del aprendizaje personal del maestro. México: Revista de Educación.

Ugarriza, N. \& Pajares, L. (2005). La evaluación de la inteligencia emocional a través del inventario de Bar-On ICE: NA, en una muestra de niños y adolescente. Lima: Persona.

Valles y Valles. (2003). Inteligencia emocional: aplicaciones educativas. España: Editorial EOS. 